\title{
Enhanced recovery care after colorectal surgery in elderly patients. Compliance and outcomes of a multicenter study from the Spanish working group on ERAS
}

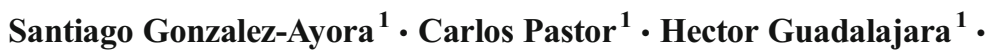 \\ Jose Manuel Ramirez ${ }^{2}$ Pablo Royo ${ }^{2}$ - Elizabeth Redondo ${ }^{2}$ - Antonio Arroyo ${ }^{3}$. \\ Pedro Moya $^{3} \cdot$ Damian Garcia-Olmo $^{1}$
}

Accepted: 20 June 2016 /Published online: 4 July 2016

(C) The Author(s) 2016. This article is published with open access at Springerlink.com

\begin{abstract}
Purpose ERAS (enhanced recovery after surgery) programs have proven to reduce morbidity and hospital stay in colorectal surgery. However, the feasibility of these programs in elderly patients has been questioned. The aim of this study is to assess the implementation and outcomes of an ERAS program for colorectal cancer in elderly patients.

Methods This is a multicenter observational study of a cohort of elderly patients undergoing colorectal surgery within an ERAS program. A total of 188 consecutive patients over 70 years who underwent elective colorectal surgery within an ERAS program at three institutions during a 2-year period were included. The compliance with the ERAS protocol interventions was measure. Complications were evaluated according to Clavien-Dindo classification. Data on length of stay and readmission rates were analyzed.

Results Early intake and early mobilization were the most successfully carried out interventions. There was a global compliance rate of $56 \%$ of patients for whom compliance was achieved with all measured interventions. The median hospital length of stay was 6 days. Almost $60 \%$ of patients had no complications, $24 \%$ had minor complications while
\end{abstract}

Santiago Gonzalez-Ayora

sgayora@fjd.es

1 Department of General Surgery, Division of Colorectal Surgery, Fundacion Jimenez-Diaz, Reyes Catolicos Ave \#2, 28040 Madrid, Spain

2 Department of General Surgery, Division of Colorectal Surgery, Hospital Clinico Universitario, Zaragoza, Spain

3 Department of General Surgery, Division of Colorectal Surgery, Hospital General Universitario, Elche, Spain
$13 \%$ had major complications; of them, $8 \%$ patients were reoperated. The readmission rate was $6.4 \%$.

Conclusions ERAS after colorectal surgery in elderly patients presents as safe and feasible based on good reported outcomes of compliance rates, complications, readmissions, and needs for reoperation.

Keywords ERAS · Enhanced · Colorectal cancer · Elderly

\section{Introduction}

Over the last decade, the enhanced recovery after surgery (ERAS) programs have generated a true revolution in colorectal surgery. This revolution has been compared to the innovative concept of total mesorectal excision for rectal surgery [1] or to that of the adoption of laparoscopic surgery as the gold standard in colon surgery $[2,3]$.

Initially proposed by Professor Henrik Khelet from Denmark, ERAS is a multidisciplinary set of care interventions in patients with the goal to obtain a comprehensive recovery after any surgical intervention $[4,5]$. Focused on colorectal cancer surgery, there exist to date strong evidence demonstrating that adhesion to ERAS protocols can minimize morbidity by decreasing secondary complications while also being cost-effective in shortening the length of hospital stay (LOS) [6-11]. Along with all the benefits of ERAS, it is known that there are significant limitations in implementing these protocols, due to the fact that compliance of all interventions may be difficult to achieve by each patient, which may worsen end results $[12,13]$. Therefore, most studies have excluded by definition elderly patients from ERAS pathways. The reason for such exclusion was that adherence to protocols in elderly patients was assumed to be unfeasible due to 
physical impairments or associated comorbidities [14]. However, currently over $70 \%$ of colorectal cancers are primarily diagnosed among patients over the age of 65 [15], and it seems reasonable that ERAS should be targeting elderly patients with associated comorbidities rather than young, healthy patients. A recent systematic review from the UK concludes that there currently exists a lack of evidence regarding ERAS in elderly patients and further studies are required to assess the implementation grade and possible benefits of ERAS protocols in this particular population [13].

This led to the design of the present multicenter study to test the hypothesis of whether it is feasible to implement an ERAS program in elderly patients undergoing elective, colorectal surgery by assessing compliance with interventions and postoperative outcomes.

\section{Materials and methods}

A retrospective analysis was performed from the GERM Group (Spanish for Grupo Español de Rehabilitacion Multimodal) prospective database by selecting patients $\geq 70$ years, undergoing colon and rectal surgery following ERAS protocols in the last 2 years (2013-2014). Data was obtained from three university tertiary centers in Spain, Hospital Universitario Fundacion Jimenez-Diaz (Madrid), Hospital Clinico Universitario (Zaragoza), and Hospital General Universitario (Elche), of consecutive patients with elective colon and rectal resections excluding urgent and palliative surgeries. A multidisciplinary task force composed of surgeons, anesthesiologist, nurses, and physical therapists was convened in each center at the beginning of the study to implement and monitor compliance with interventions. A nurse coordinator was assigned to each center to routinely assess ERAS compliance obtained from the patient's medical records and to report monthly the results to the study committee. In addition, a videoconference meeting was held every 3 months involving all three centers. The study was initiated after obtaining approval by the local institutional review board committee from each institution.

A bundle of 10 interventions were adopted at the same time in all three participating hospitals on the basis of our previously published protocol $[16,17]$ including the following: (1) preoperative advice and evaluation of nutritional status, (2) intravenous iron supplementation in cases of preoperative anemia, (3) avoidance of full mechanical preparation for colon resections, (4) administration of carbohydrate-rich drinks 1 day prior and on the morning of surgery, (5) goal-directed intraoperative fluid therapy and body temperature control during surgery, (6) the use of intraoperative pneumatic legs compression, (7) avoidance of nasogastric tubes and drains when possible, (8) taking in oral fluids during the early postoperative period (means $6-8 \mathrm{~h}$ after surgery) and soft-food diet by the second postoperative day and early mobilization (walking from the bed to the sofa at 6-8 $\mathrm{h}$ after surgery), (9) intravenous fluid restriction and removal of urinary catheter (indicates stopping fluids and catheter removal by the first postoperative day), and (10) multimodal analgesia (epidural catheter for open surgery cases).

By definition, we targeted hospital discharge date at fourth or fifth postoperative day for colon or rectal surgery, respectively. Patients could be discharged if they met the following criteria: good mobilization, adequate oral intake for liquids and solids, recovered gastrointestinal transit minimally for passing gas, normal urinary outputs, no wound problems, good pain control with oral medication, absence of fever in the last $48 \mathrm{~h}, \mathrm{C}$-reactive protein below $10 \mathrm{mg} / \mathrm{L}$ at discharge, and showing a decreasing trend in previous laboratory test. In addition, the patient and family needed to feel comfortable with the discharge and information given regarding possible complications and early detection.

Compliance with all interventions was combined and expressed as the percentage of patients who had correct intervention and documentation. Global compliance (GC) was defined as the rate of patients for whom compliance was achieved with all measures of the ERAS protocol.

The POSSUM score (Physiological and Operative Severity Score for the enumeration of Mortality and Morbidity) [18] was determined to calculate postoperative morbidity and mortality risks. Additional operative variables such as a stoma construction and the use of a laparoscopic surgical approach were also recorded. Short-term postoperative complications were graded using the Clavien-Dindo classification [15]. LOS and rates and causes of readmission during the first 30day postoperative period were also documented. A further analysis was performed when LOS was prolonged to identify the causes of delayed hospital discharge.

\section{Statistical analysis}

Patient baseline characteristics at the time of surgery, age, gender, American Society of Anesthesiologists (ASA) score, major comorbidities, preoperative anticoagulation therapy were obtained from each patient's electronic medical record. Compliance information was gathered from inpatient medical charts and recorded on a monthly basis in the database. Any measure during the process was considered as "noncompliant" if documentation was incomplete. Descriptive statistics are presented using $t$ test with mean and standard deviation (SD) or median and range for continuous variables. Comparison of differences between group means was carried out using ANOVA for variables with normal distribution, and the Mann-Whitney test for continuous variables with nonparametric distribution. We used chi-squared analysis with Fisher's exact test when any value observed in the contingency table was less than 5 to compare proportion variables. We 
performed a Kruskal-Wallis test to explore the impact of compliance within interventions on the length of stay. The level of statistical significance was taken as $p<0.05$. All statistical calculations were performed using SPSS software ${ }^{\circledR}$ (version 22.0, SPSS Inc., Chicago, IL).

\section{Results}

Throughout the study period a total of 188 patients were treated among the three centers. Of them, 109 (58\%) were men, 79 $(42 \%)$ were women with a median age for the whole group of 79 [70-93] years old. Demographics, patient baseline characteristics, and surgical procedures are presented in Table 1. It is important to note that sigmoid resection and right and left colectomies were the most common surgery; $77 \%(n=145)$ performing a primary anastomosis. We also included rectal cancer surgeries $(n=43,23 \%)$; of them, 34 patients in whom low anterior resections were done with or without a temporary stoma. By summarizing these cases, $95 \%$ of patients had a primary anastomosis with sphincter preservation. In addition, almost $45 \%$ of surgeries ( $40 \%$ colectomies; $58 \%$ rectal surgeries) were performed via laparoscopic approach.

Table 1 Patient baseline characteristics and surgical techniques

\begin{tabular}{|c|c|c|}
\hline Variable & $n=188$ & \\
\hline Age (years) ${ }^{\mathrm{a}}$ & 79 & {$[70-93]$} \\
\hline Male & 109 & $58 \%$ \\
\hline Female & 79 & $42 \%$ \\
\hline POSSUM score (morbidity) ${ }^{\mathrm{a}}$ & $29.3 \%$ & [6.9-80.5] \\
\hline POSSUM score (mortality) ${ }^{\mathrm{a}}$ & $5.3 \%$ & {$[1.3-26.8]$} \\
\hline \multicolumn{3}{|l|}{ Comorbidities } \\
\hline Diabetes & 52 & $28 \%$ \\
\hline Anticoagulant treatments & 36 & $19 \%$ \\
\hline \multicolumn{3}{|l|}{ Surgical technique } \\
\hline Colon surgery & 145 & $77.1 \%$ \\
\hline Laparoscopic colon surgery & 58 & $40.0 \%$ \\
\hline Rectal surgery & 43 & $22.9 \%$ \\
\hline Laparoscopic rectal surgery & 25 & $58.1 \%$ \\
\hline Total laparoscopic surgery & 83 & $44.1 \%$ \\
\hline \multicolumn{3}{|l|}{ Surgical procedure } \\
\hline Right colectomy & 80 & $42.6 \%$ \\
\hline Left colectomy & 15 & $8.0 \%$ \\
\hline Sigmoidectomy & 50 & $26.6 \%$ \\
\hline Low anterior resection & 34 & $18.1 \%$ \\
\hline Hartmann & 7 & $3.7 \%$ \\
\hline Abdominoperineal resections & 2 & $1.1 \%$ \\
\hline Stoma (temporary or definitive) & 25 & $13.3 \%$ \\
\hline
\end{tabular}

${ }^{\mathrm{a}}$ Median [range]
The POSSUM scoring system was calculated for each patient immediately after the surgery, with a median of $29.3 \%$ [6.9-80.5] and 5.3\% [1.3-26.8] for expected postoperative morbidity and mortality, respectively.

\section{Compliance outcomes}

Data was measured independently for each ERAS intervention as is presented in Table 2. By definition, we avoided mechanical preparation in colonic surgeries, and all patients received preoperative dietary recommendations and a carbohydrate-rich drink at 2-4 h before surgery. Early intake of clear liquids at $6 \mathrm{~h}$ after surgery and early mobilization were the most successfully carried out interventions in over $90 \%$ of patients. On the other hand, the discontinuing early of intravenous fluids and early removal of urinary catheter rates were 73 and $64 \%$, respectively.

Overall, there was a GC rate of $56 \%$ of patients for whom compliance was achieved with all measured interventions. When performing laparoscopic surgery a higher, but nonsignificant GC percentage was achieved (59\% lap vs. $53.3 \%$ open; $p=0.43$ ). Patients who underwent rectal surgery had significantly lower $\mathrm{GC}$ rates when compared to colon surgery ( $41.9 \%$ rectal vs. $60.0 \%$ colon; $p=0.03)$. In addition, a construction of either a temporary or a definitive stoma, also decreased GC rates $(36.0 \%$ with stoma vs. $58.9 \%$ without stoma; $p=0.03$ ).

\section{Postoperative outcomes}

Postoperative outcomes, surgical complications, and length of stay are showed in Table 3. About $60 \%$ of patients had an

Table 2 Compliance rates with ERAS interventions

\begin{tabular}{|c|c|c|}
\hline \multicolumn{2}{|l|}{ Variable } & $n(\%)$ \\
\hline \multicolumn{2}{|l|}{ No drainage ${ }^{a}$} & $81(43.0)$ \\
\hline \multicolumn{2}{|l|}{ Epidural anesthesia $^{\mathrm{a}}$} & $116(61.7)$ \\
\hline \multicolumn{2}{|l|}{ Early intake } & $173(92.0)$ \\
\hline \multicolumn{2}{|c|}{ Early suspension of intravenous fluids } & $138(73.4)$ \\
\hline \multicolumn{2}{|l|}{ Early mobilization } & $169(89.9)$ \\
\hline \multicolumn{2}{|c|}{ Early urinary catheter removal } & $122(64.9)$ \\
\hline \multicolumn{2}{|l|}{ Global compliance } & $105(56.0)$ \\
\hline Variable & Global compliance $(\%)$ & $X^{2} p$ value \\
\hline Laparoscopic surgery & 59.0 & 0.43 \\
\hline Open surgery & 53.3 & \\
\hline Colon surgery & 60.0 & 0.03 \\
\hline Rectal surgery & 41.9 & \\
\hline Without stoma ${ }^{\mathrm{b}}$ & 58.9 & 0.03 \\
\hline With stoma ${ }^{\mathrm{b}}$ & 36.0 & \\
\hline
\end{tabular}

${ }^{\text {a }}$ ERAS interventions not included in the global compliance

${ }^{\mathrm{b}}$ Temporary or definitive stoma 
uneventful postoperative course without complications (66.7 \% when GC achieved vs. $56.6 \%$ without GC), $25 \%$ had minor complications, prolonged ileus being the most common complication. Important to note, we did not observe frequent medical complications in elderly patients such as postoperative pneumonia or venous thromboembolism. Major complications occurred in $13 \%$ of cases, including 15 patients who had a clinical anastomotic leakage (8\%). The need for a reoperation was $8.5 \%$ (16 patients) mainly due to anastomotic leakage. Mortality following complications occurred in three patients $(1.6 \%)$. As expected, patients who had rectal surgeries suffered worse complications compared to patients with colon surgeries and with a higher risk of anastomotic leakage.

When investigating the impact of GC on postoperative complications, we observed the following data as shown in Table 3: (1) a clinical, but not statistically significant $10 \%$ reduction on minor Clavien-Dindo I/II complications (30\% with GC vs. $20 \%$ without GC, $p=0.32$ ). While we did not observed influences of achieving GC on major Clavien-Dindo III/IV complications (13.4 \% with GC vs. $13.2 \%$ without, $p=0.34)$, (2), a lower percentage of postoperative ileus (21\% with GC vs. $26.5 \%$ without GC; $p=0.37$ ), and (3) a

Table 3 Postoperative outcomes

\begin{tabular}{lll}
\hline Postoperative complications & $n(\%)$ & $X^{2} p$ value \\
\hline No complications & $117(62.2)$ & \\
Dindo-Clavien grades I-II & $46(24.5)$ & 0.32 \\
& with GC 30 & \\
without GC 20 & 0.34 \\
Dindo-Clavien grades III-V & $25(13.3)$ & \\
& with GC 13.4 & \\
Postoperative ileus & without GC 13.2 & \\
& $44(23.4)$ & \\
& with GC 21.0 & \\
Anastomotic leakage & without GC 26.5 \\
& $15(8.0)$ & \\
Mortality & with GC 7.6 & \\
Reoperations & without GC 8.4 & \\
Anastomotic leakage & $3(1.6)$ & \\
Bowel ischemia & $16(8.5)$ & \\
Hemoperitoneum & $10(5.3)$ & \\
Internal hernia & $2(1.0)$ & \\
Urinary tract injury & $1(0.5)$ & \\
Hospital length of stay & $1(0.5)$ & \\
& $1(0.5)$ & \\
Readmissions & $6.0[3-51]$ & \\
Abdominal abscess & with GC 6 [3-30] & \\
Wound infection & without GC 7 [4-51] & \\
Late anastomotic leakage & $3(1.6)$ & \\
Dehydration & $3(1.6)$ & \\
\hline
\end{tabular}

${ }^{\mathrm{a}}$ Median [range] non-significant effect of GC in terms of anastomotic leakage was present ( $7.6 \%$ with GC vs. $8.4 \%$ without GC; $p=0.83$ ).

Median LOS was 6 [3-51] days for the entire study population including the readmission days when occurred. A significant impact of GC on LOS was observed with a median reduction of 1 day in LOS including readmissions (6 [3-30] days with GC vs. 7 [4-51] days without GC; $p=0.03$ ).Figure 1 shows the influence of percentage of GC in reduction of median LOS. A significant reduction of 1.5 days in LOS was achieved when we were able to reach a $50 \%$ rate in $\mathrm{GC}(7.5[5-51]$ days $<50 \% \mathrm{GC}$ vs. 6 days [3-30] days $>50 \% ; p<0.04$ ]Figure 2 shows further analysis of LOS based on the postoperative day, showing that $41 \%$ of the study population were discharged at ERAS or ERAS + 1 day periods. The main reasons for a delayed discharge are shown in Fig. 2. indicating social issues to be the most observed reasons for delay in discharge compared to postoperative complications. Readmission to the hospital occurred in 12 patients $(6.4 \%)$. The most common cause of readmission was deep and organ/ space (intra-abdominal) infections requiring CT-guided percutaneous drainage and/or antibiotic coverage. There were two cases of late anastomotic leakage with pelvic abscesses in patients who underwent low anterior resections

\section{Discussion}

Our data shows reliable outcomes from a multicenter, observational, non-randomized study group in the implementation of a standardized ERAS protocol in elective colorectal surgery in elderly patients.

There is a lack of information on the exact impact of ERAS interventions in elderly patients, although the current evidenced-based data has been recently reviewed in a systematic review from the UK [13]. This review reported to date two clinical trials comparing ERAS with non-ERAS, focused on elderly patients showing in favor of ERAS, a shorter length of stay and a significant decrease in minor complications [19, 20]. However, one of these studies had a low number of patients, did not show data about rates of reoperation or readmission, and did not report compliance with interventions. Due to lacking such data, our study proved that a sustained effort from a dedicated multidisciplinary team could achieve a high level of compliance rates with most ERAS interventions in both colon and rectal surgeries focused in elderly patients.

When analyzing compliance with ERAS bundles, we obtained the goal of $90 \%$ compliance within two interventions: early intake of clear liquids and early mobilization when planned. Both were difficult to be implemented and required an extra effort from the caregivers, as most elderly patients were reluctant to get out of bed or drink liquids $6-8 \mathrm{~h}$ after surgery. In our experience, management of proper pain control within over $60 \%$ of patients with epidural morphine-sparing analgesia can help to accomplish early mobility from the bed 
Fig. 1 Influence of Compliance with ERAS measures in LOS (Kruskal-Wallis test)

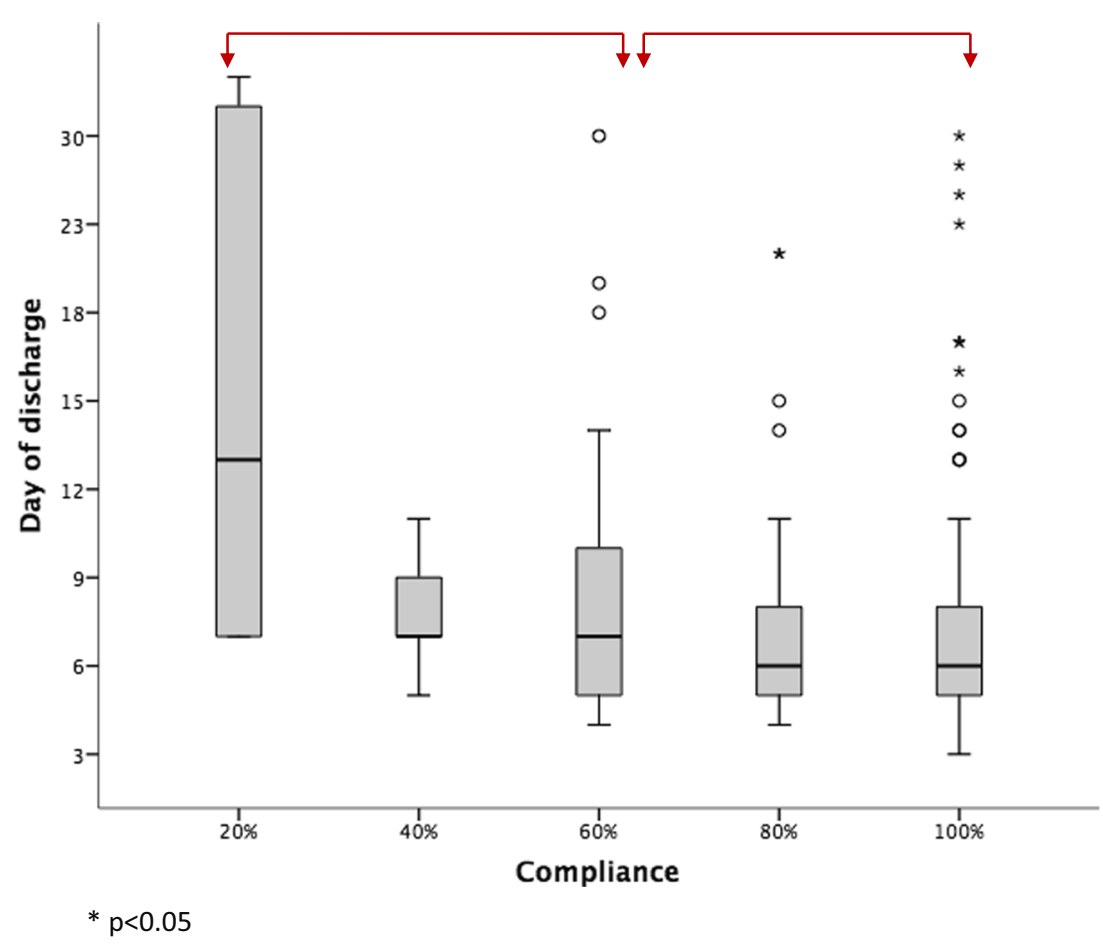

$<50 \%$ of GC LOS: 7.5 days [5-51] vs. $>50 \%$ of GC LOS: 6 days [3-30]; $p=0.004$ to the sofa without adding secondary effects. Moreover, almost half of the participants where operated via laparoscopic approach, adding advantages in achieving these compliance rates. From the rest of ERAS interventions, early stopping of intravenous fluids and early urinary catheter removal showed the lowest compliance rates ( 73 and $65 \%$, respectively). We believe the reason for this delay was due to short urinary outputs in elderly patients during the first postoperative day leading to a delayed removal of the urinary catheter. Instead of looking at each specific intervention, we developed the variable global compliance, defined as the rate of patients for whom compliance was achieved with all the measurements of the ERAS protocol. Overall, there was a GC rate of $56 \%$ in the study population. Identified barriers to achieve a higher
Fig. 2 Reasons for delaying discharge beyond ERAS estimated date. *Estimated discharge in ERAS protocol

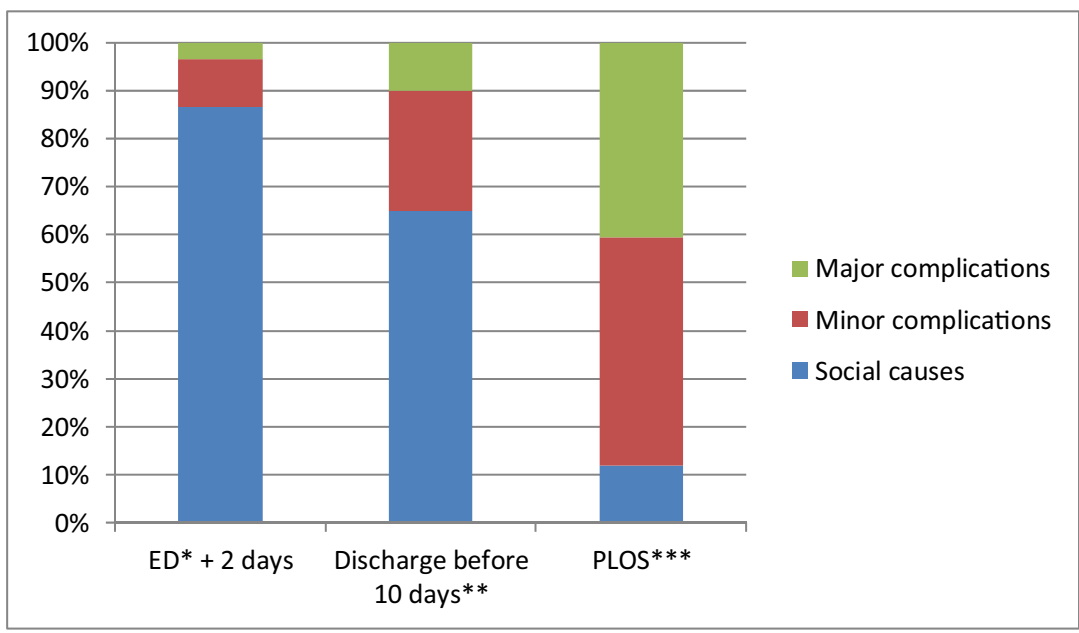

* Estimated discharge in ERAS protocol.

** Discharge before 10 days excluding ED / ED + 1 / ED + 2 .

$* * *$ Prolonged length of stay (discharge $\geq 10$ days). 
GC with statistical differences were rectal surgeries, the creation of a stoma and open surgery cases. Therefore, a great effort should be made to increase our plan of care in patients with these characteristics.

Criticism of ERAS protocols will argue that a high readmission rate especially in elderly patients will invalidate any positive result. Regarding 30-day postoperative outcomes, our data showed that $62 \%$ of patients had no complications, $25 \%$ had minor complications, and $13 \%$ suffered major ClavienDindo's complications. Postoperative ileus was the most common observed complication in almost $25 \%$ of patients who required a nasogastric tube and prolonged total parenteral nutrition. We did not observe respiratory complications such as pneumonia or pulmonary edema or cases of deep vein thrombosis. Reoperation was needed in $8.5 \%$ of patients and clinical anastomotic leakage occurred in $8 \%$ for colon surgeries and $11.6 \%$ in rectal surgeries.

An impact of GC on decreasing postoperative complications has been earlier reported in 2011 by a multicenter study from the European ERAS Study Group especially when GC could be achieved by over $70 \%$ [21]. When analyzing the impact of GC in postoperative complications, there was a clinical, but not significant reduction of $10 \%$ in minor complications and about 5-6\% in postoperative ileus, while mayor postoperative complications such as anastomotic leakage remained unchanged. Our aim was to establish and detect an impact of GC in decreasing complications; however, a small sample size may have underpowered the effect of GC in complications in our study.

For predicting patients at risk of developing complications, we assessed for each patient their POSSUM score. We believed that the POSSUM score might be more useful than the ASA score to predict postoperative outcomes and that it is adequate to assess patient's baseline performance status; however, it is not a valid tool to identify patients at risk of failure in the ERAS programs. The key point would be to select in the preoperative evaluation who will fail in achieving ERAS, to either design a personalized program for those patients, or have the opportunity to improve their conditions in the prehabilitation period. In this sense, a better score taking into account "frailty" rather than "elderly" using the modified frailty index has demonstrated to correlate better with complications, longer lengths of stay, and readmissions and has recently been validated in elderly patients undergoing colorectal surgery under ERAS protocols [22]. Therefore, we would recommended before implementing ERAS in elderly patients to use a prospective score to identify patients at risk for not achieving the protocol so resources and postoperative supports would be better allocated.

Contemporary postoperative admission stays in ERAS protocols range from 3 to 5 days [23] in comparison to traditional practice of up to 5 to 9 days. Focused on surgery in elderly patients, a prospective study of 87 patients $>70$ years old, reported a mean LOS of 3.9 days [24]. LOS is often used as a surrogate marker of recovery, and it should not be offset by a higher rate of hospital readmission.

In our data, the median LOS was 6 days for the entire study population with minimal differences between hospitals. When analyzing LOS by subgroups, $41 \%$ of patients were discharge at the estimated day in ERAS protocols (in the fourth postoperative day for colon surgery or fifth postoperative day for rectal surgery) or ERAS + 1 day. Moreover, when analyzing the reasons for a delayed discharge, a needed of social support and non-postoperative complications were the most common causes for patients to be discharged from the hospital before the 10th postoperative day. We believe this to be because two of the hospitals from the study are reference hospitals covering suburban and rural areas. These patients may live a greater distance from the hospital, making physicians reluctant to discharge them earlier. Important to note, our data showed a positive effect of GC in LOS when we were able to achieve, at least, $50 \%$ of compliance with the interventions.

Readmission to the hospital after discharge was observed in $6.4 \%$ of patients, mainly due to abdominal abscess after pelvic surgeries. We reported two cases of late anastomotic leakage in rectal cancer surgeries in patients who were readmitted to the hospital needing percutaneous drainage. In contrast, there were no cases of delayed leakage in colon resections. We believe that, based on the study population of elderly patients, these LOS and readmissions rates are considerably good and support the idea that ERAS is a feasible and secure option for this particular population.

Our study has some limitations that deserve to be mentioned. First, although this was a multicenter study, it was not being conducted, as a randomized clinical trial, and the number of cases was small in order to established robust conclusions. Secondly, we did not compare our results in elderly patients to a control group of patients $<70$ years old with the same ERAS protocol or under the traditional treatment, due to a lack of information in our previous database prior to the start of the study.

\section{Conclusions}

Based on our data from the present multicenter study, ERAS should be implemented without reservations in elderly patients undergoing elective colorectal surgeries, expecting the same goals and benefits as found in other age groups. Barriers in achieving a high compliance with ERAS interventions in elderly patients are common and will require a great effort in the patient education, an intensive plan of pre and postoperative care, and sometimes a change in the surgeons' management. 
Acknowledgments The authors thank Sara Rosenstone Calvo and Patricia Tejedor M.D. for editing this manuscript.

Author's contributions Santiago Gonzalez-Ayora and Carlos Pastor contributed equally to analyze the data and write the manuscript.

\section{Compliance with ethical standards}

Conflict of interest The authors declare that they have no conflict of interest.

Open Access This article is distributed under the terms of the Creative Commons Attribution 4.0 International License (http:// creativecommons.org/licenses/by/4.0/), which permits unrestricted use, distribution, and reproduction in any medium, provided you give appropriate credit to the original author(s) and the source, provide a link to the Creative Commons license, and indicate if changes were made.

\section{References}

1. Heald RJ, Husband EM, Ryall RD (1982) The mesorectum in rectal cancer surgery - the clue to pelvic recurrence? Br J Surg 69:613-616

2. Biondi A, Grosso G, Mistretta A, Marventano S, Toscano C, Drago F, Gangi S, Basile F (2013) Laparoscopic vs. open approach for colorectal cancer: evolution over time of minimal invasive surgery. BMC Surg 13(Suppl 2):S12

3. Morneau M, Boulanger J, Charlebois P, Latulippe JF, Lougnarath R, Thibault C, Gervais N (2013) Laparoscopic versus open surgery for the treatment of colorectal cancer: a literature review and recommendations from the Comite de l'evolution des pratiques en oncologie. Can J Surg J Canadien de Chirurgie 56:297-310

4. Kehlet H, Wilmore DW (2002) Multimodal strategies to improve surgical outcome. Am J Surg 183:630-641

5. Kehlet H, Wilmore DW (2005) Fast-track surgery. Br J Surg 92:3-4

6. Wind J, Polle SW, Fung Kon Jin PH, Dejong CH, von Meyenfeldt MF, Ubbink DT, Gouma DJ, Bemelman WA (2006) Systematic review of enhanced recovery programmes in colonic surgery. Br J Surg 93:800-809

7. Adamina M, Kehlet H, Tomlinson GA, Senagore AJ, Delaney CP (2011) Enhanced recovery pathways optimize health outcomes and resource utilization: a meta-analysis of randomized controlled trials in colorectal surgery. Surgery 149:830-840

8. Spanjersberg WR, Reurings J, Keus F, van Laarhoven CJHM (2011) Fast track surgery versus conventional recovery strategies for colorectal surgery (review). The Cochrane Library Issue 2

9. Odermatt M, Miskovic D, Flashman K, Khan J, Senapati A, O'Leary D, Thompson M, Parvaiz A (2015) Major postoperative complications following elective resection for colorectal cancer decrease long-term survival but not the time to recurrence. Color Dis Feb. 17(2):141-149.

10. Liang LV, Sao Y-f, Zhou Y-b (2012) The enhanced recovery after surgery (ERAS) pathway for patients undergoing colorectal surgery: an update of meta-analysis of randomized controlled trials. Int J Color Dis 27:1549-1554

11. Lohsiriwat V (2014) Impact of an enhanced recovery program on colorectal cancer surgery. Asian Pac J Cancer Prev 15:3825-3828

12. Polle SW, Wind J, Fuhring JW, Hofland J, Gouma DJ, Bemelman WA (2007) Implementation of a fast-track perioperative care program: what are the difficulties? Dig Surg 24:441-449

13. Bagnall NM, Malietzis G, Kennedy RH, Athanasiou T, Faiz O, Darzi A (2014) A systematic review of enhanced recovery care after colorectal surgery in elderly patients. Color Dis 16:947-956

14. Pawa N, Cathcart PL, Arulampalam THA, Tutton MG, Motson RW (2012) Enhanced recovery program following colorectal resection in eldery patient. World J Surg 36:415-423

15. Dindo D, Demartines N, Clavien PA (2004) Classification of surgical complications: a new proposal with evaluation in a cohort of 6336 patients and results of a survey. Ann Surg 240:205-213

16. Ramirez JM, Blasco JA, Roig JV, Maeso-Martinez S, Casal JE, Esteban F, Lic DC (2011) Enhanced recovery in colorectal surgery: a multicentre study. BMC Surg 11:9

17. Esteban F, Cerdan FJ, Garcia-Alonso M, Sanz-Lopez R, Arroyo A, Ramirez JM, Moreno C, Morales R, Navarro A, Fuentes M (2013) A multicentre comparison of a fast track or conventional postoperative protocol following laparoscopic or open elective surgery for colorectal cancer surgery. Color Dis 16:134-140

18. Copeland GP, Jones D, Walters M (1991) POSSUM: a scoring system for surgical audit. Br J Surg 78:355-360

19. Wang Q, Suo J, Jiang J, Wang C, Zhao YQ, Cao X (2012) Effectiveness of fast-track rehabilitation vs conventional care in laparoscopic colorectal resection for elderly patients: a randomized trial. Color Dis 14:1009-1013

20. Jia Y, Jin G, Guo S, Gu B, Jin Z, Gao X, Li Z (2014) Fast-track surgery decreases the incidence of postoperative delirium and other complications in elderly patients with colorectal carcinoma. Langenbeck's Arch Surg / Deutsche Gesellschaft fur Chirurgie 399:77-84

21. Gustafsson UO, Hausel J, Thorell A, et al. (2011) Adherence to the enhanced recovery after surgery protocol and outcomes after colorectal cancer surgery. Arch Surg 146(5):571-577

22. Keller DS, Bankwitz B, Nobel T, Delaney CP (2014) Using frailty to predict who will fail early discharge after laparoscopic colorectal surgery with an established recovery pathway. Dis Colon rectum. Mar 57(3):337-342.

23. DiFronzo LA, Yamin N, Patel K (2003) Benefits of early feeding and early hospital discharge in elderly patients undergoing open colon resection. J Am Coll Surg 197:747

24. DiFronzo LA, Yamin N, Patel K (2003) Benefits of early feeding and early hospital discharge in elderly patients undergoing open colon resection. J Am Coll Surg 197:747 